\title{
Gastrointestinal cancers: the role of microbiota in carcinogenesis and the role of probiotics and microbiota in anti-cancer therapy efficacy
}

\author{
KAROLINA KAŹMIERCZAK-SIEDLECKA ${ }^{1}$, JAKUB RUSZKOWSKI ${ }^{2,3}$, \\ KAROLINA SKONIECZNA-ŻYDECKA4, JAKUB JĘDRZEJCZAK ${ }^{5}$, MARCIN FOLWARSKI ${ }^{6}$, \\ WOJCIECH MAKAREWICZ ${ }^{1}$ \\ ${ }^{1}$ Department of Surgical Oncology, Medical University of Gdansk, Gdansk, Poland \\ ${ }^{2}$ Department of Physiopathology, Medical University of Gdansk, Gdansk, Poland \\ ${ }^{3}$ Department of Nephrology, Transplantology and Internal Diseases, Medical University of Gdansk, Gdansk, Poland \\ ${ }^{4}$ Department of Biochemistry and Human Nutrition, Pomeranian Medical University, Szczecin, Poland \\ ${ }^{5}$ Student Research Group at the Department of Inorganic Chemistry, Medical University of Gdansk, Gdansk, Poland \\ ${ }^{6}$ Department of Clinical Nutrition and Dietetics, Medical University of Gdansk, Gdansk, Poland
}

\begin{abstract}
The gut epithelium is a habitat of a variety of microorganisms, including bacteria, fungi, viruses and Archaea. With the advent of sophisticated molecular techniques and bioinformatics tools, more information on the composition and thus function of gut microbiota was revealed. The gut microbiota as an integral part of the intestinal barrier has been shown to be involved in shaping the mucosal innate and adaptive immune response and to provide protection against pathogens. Consequently, a set of biochemical signals exchanged within microbes and communication between the microbiota and the host have opened a new way of thinking about cancer biology. Probiotics are living organisms which administered in adequate amounts may bring health benefits and have the potential to be an integral part of the prevention/treatment strategies in clinical approaches. Here we provide a comprehensive review of data linking gut microbiota to cancer pathogenesis and its clinical course. We focus on gastrointestinal cancers, such as gastric, colorectal, pancreatic and liver cancer.
\end{abstract}

Key words: gastrointestinal microbiome, carcinogenesis, probiotics, dysbiosis.

(Centr Eur J Immunol 2020; 45 (4): 476-487)

\section{Introduction}

A complex of intestinal microorganisms, referred to as gut microbiota, has recently been acknowledged as one of the pivotal players of human health [1]. Multiple studies have elegantly demonstrated that gnotobiotic models present skewed biochemical traits, at least partly restored via recolonization and/or administration of microbiota metabolites [2, 3]. Indeed, fecal transplantation has been shown to induce long-term immunity-related effects positively associated with carcinogenesis secondary to infection [4]. Overall, the involvement of altered gut microbiota in tumorigenesis of both local and distal tissues is considered to be predominantly a result of disrupted immune-related pathways [5]. In particular, microbiota can induce tumor promoting inflammation, as well as the ability to evoke immunosuppression that helps cancer cells to avoid immune destruction [6]. Additionally, microbial metabolites can deregulate host genome stability preceding cancer development [6]. The most common mechanism in which microbiota alterations may be involved in cancer pathophysiology is presented in Figure 1. Below, we review both mechanisms in the context of gastrointestinal cancers.

Beyond the participation in carcinogenesis, indigenous microbiota can play a pivotal role in anti-cancer treatment. The very first study proving the immunomodulatory effect of gut microbiota on oncogenesis provided evidence that total body irradiation elevated the passage of lipopolysaccharide by tumor-specific $\mathrm{T}$ cells from the gut lumen to lymphoid organs. Additionally, dendritic cells were found to be more intensively activated and the systemic concentration of proinflammatory biomarkers was found to be elevated [7]. It was shown that the administration of microbial ligand-containing serum or ultrapure lipopolysaccharide (LPS) from irradiated mice to nonirradiated counterparts (antibody-lymphodepleted) enhanced $\mathrm{CD}^{+} \mathrm{T}$ cell activation and improved regression of tumor. Moreover, the administration of ultrapure LPS to irradiated mice increased 


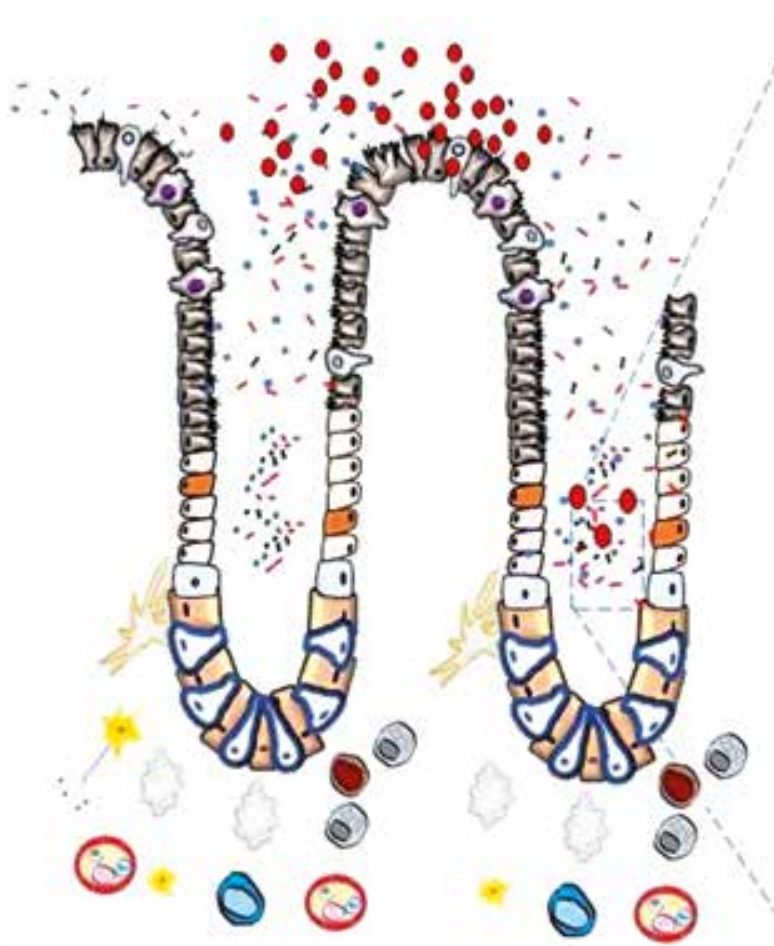

Cancer-associated pathogenic bacteria

Helicobacter pylori

- E-cadherin/ $\beta$-catenin signaling

- Autophagy inhibition

- Oxidative damage enhancement

- Increased expression of Bcl-xL, MCL-1, survivin, c-myc, cyclin D-1

Helicobacter hepaticus

- increased p21 expression

Peptostreptococcus anaerobius

- Increased expression of SREBP-2 and AMPK

Fusobacterium nucleatum

- E-cadherin/ $\beta$-catenin signaling

- NK cell cytotoxity inhibition

\section{Dysbiosis}

- Elevated intestinal permeability

- TLRs activation by MAMPs (TLR4 promoting hepatocellular and pancreatic cancer)

- Pro-inflammatory cytokine synthesis

- Inflammasome activation and autophagy induced via TLRs

- NF- $\kappa \mathrm{B}$ and STAT3 signaling pathway activation

- Genomic instability

- Skewed inflammatory response of the host

Fig. 1. Selected microbiota-dependent mechanisms involved in carcinogenesis

the number and function of the adoptively transferred cells and as a consequence led to long-term cure of mice with large B16F10 tumors. The evidence was followed by a series of studies confirming that cancer treatment is less effective when administered to germ-free animals or rodents treated with antibiotics [8]. Microbial alterations are linked to these associations, being responsible for elevated intestinal permeability and thus lower gut barrier integrity. For instance, it was shown that cyclophosphamide induced dysbiosis and initiated the translocation of Enterococcus hirae into the spleen or Barnesiella intestinihominis into the colon, thus mediating immunological processes of anti-tumor response. This led to an increased intratumoral CD8/Treg ratio and promotion of infiltration of IFN- $\gamma$-producing $\gamma \delta \mathrm{T}$ cells in cancer lesions, respectively [9].

Interestingly, recent data support the hypothesis that certain gut microbiota composition may influence the anti-cancer treatment efficacy [10]. Using different "-omic" approaches, scientists could stratify patients as responders and non-responders. This is what has recently been done by means of metagenomic fingerprints in persons treated with anti-PD-1 antibodies [11-14]. Overall, the higher abundance of commensal microbes, such as Clostridiales, Ruminococcaceae, Faecalibacterium spp., Akkermansia muciniphila, Bacteroides fragilis, Bifidobacteria, Enterococci, Collinsella, and Alistipes may elevate the probability of higher treatment success [15]. If so, studies suggest that certain probiotics administered to restore homeostasis within gut microbiota may modify the risk of carcinogenesis. Furthermore, microbiota-based therapy has the potential to enhance the anti-cancer treatment and diminish the prevalence of adverse effects [16-18]. Lastly, gut bacteria were reported to be involved in deactivating drugs that target cancer cells.

\section{Gastric cancer}

Nowadays, gastric cancer is the fourth most common type of cancer worldwide [17]. The stomach is also a site of the most common marginal zone lymphoma of the digestive tract - gastric mucosa-associated lymphoid tissue (MALT) lymphoma [19]. One of the preventable risk factors of both gastric cancer and gastric MALT lymphoma is Helicobacter pylori infection [20]. This Gram-negative bacterium possesses several mechanisms to survive in the acid gastric lumen and to induce chronic gastritis [19]. While gastric adenocarcinoma is associated with infection with certain $H$. pylori strains (possessing a cag pathogenicity island in their genomes), gastric MALT lymphoma can be a result of any H. pylori infection [19]. The eradication of $H$. pylori is recommended as a prevention and part of treatment in the abovementioned cancers [20].

Gastric microbiota mainly consists of Proteobacteria, Firmicutes, Bacteroidetes, Fusobacteria and Actinobacte- 
ria [21]. However, the composition of gastric microbiota varies individually. Gastric microbiota may be associated with gastric cancer development. Wang et al. observed that Lactobacillus, Escherichia-Shigella, Nitrospirae, Burkholderia fungorum and Lachnospiraceae were enriched in gastric mucosal biopsy specimens from patients with non-cardia gastric cancer [21]. Thus increased abundance of lactic acid bacteria (Lactococcus and Lactobacillus genera) may be linked to the carcinogenesis process. Yu et al. have reported that Lactobacillus sp. is commonly known as a genus beneficial to the host, but elevated levels of lactic acid being its common metabolite can be detrimental in the context of cancer development [22]. Lactate was shown to promote inflammation and stimulate tumor angiogenesis. Additionally, it can be a source of energy for tumor cells, because it induces glycolytic enzymes. Moreover, enrichment in short-chain fatty acids (SCFAs) (predominantly butyrate, propionate, acetate) production has also been reported. Furthermore, increased levels of bacterial SCFAs were demonstrated to induce hyperproliferation of colonic cells and oesophageal trans-differentiation in Barrett's esophagus [22, 25]. However, the major carcinogenic pathogen of the stomach is Helicobacter pylori. It initiates mucosal inflammation, leads to mucosal atrophy, and as a consequence, causes development of gastric cancer [22, 25].

Administration of probiotic bacteria which belong to Lactobacillus and Bifidobacterium genera was demonstrated to potentially decrease side effects of antibiotic therapy, which is a part of eradication therapy. It also improves efficiency of eradication and supports intestinal microbiota balance, referred to as eubiosis [23]. The meta-analysis of Tong et al., which included 14 randomized clinical trials, showed that using probiotics during antibiotic therapy increased the rate of Helicobacter pylori eradication. The authors found that such microbial treatment elevated the eradication success by almost $80 \%$ and diminished the incidence of diarrhea, being the most prevalent adverse effect of the eradication process, by almost half [16]. What is more interesting, a recent meta-analysis showed that probiotics alone may eradicate $H$. pylori in $14 \%$ of patients [24]. Even though this is an unsatisfactory rate from a clinical point of view, the results confirmed probiotics' direct antibacterial action against $H$. pylori [24]. The viable gastric microbiome is associated with $H$. pylori serological status and it is altered in gastric carcinogenesis [25]. The changes in the gastric microbiome are not only associated with abundance of $H$. pylori, but also with enriched amounts of other bacterial species. Patients with gastric cancer were demonstrated to be enriched in Lactococcus, Veillonella and Fusobacteriaceae (Fusobacterium and Leptotrichia) in comparison to control subjects (functional dyspepsia patients) [25].

Moreover, probiotics can be used to prevent side effects of perioperative nutritional support in patients with gastric cancer. Malnutrition is common in upper gastrointestinal cancer, contributing to complications of major abdominal surgery and worse overall survival [26]. However, enteral nutrition is associated with the occurrence of complications such as nausea, vomiting, flatulence, stomach ache and diarrhea [26]. Zhao et al. assessed the influence of fiber and probiotics administration on diarrheal symptoms associated with enteral nutrition in patients with gastric cancer. This trial included 120 patients who received an enteral diet for 7 days after surgery. They were divided into 3 groups: fiber-free nutrition formula $(n=40)$, fiber-enriched nutrition formula $(n=40)$, fiber- and probiotic-enriched nutrition formula $(n=40)$. It was observed that the frequency of diarrhea was higher in patients receiving the fiber-free nutrition formula compared to the fiber-enriched nutrition formula $(p=0.007)$. Moreover, patients who consumed the fiber- and probiotic-enriched formula suffered less frequently from diarrhea compared to the fiber-enriched nutrition formula $(p=0.003)$. There were no significant differences between study groups regarding laboratory parameters (prealbumin, albumin, transferrin, total lymphocyte count) on the seventh day of dietary treatment. Overall, probiotics in combination with fiber reduced the incidence of diarrhea, which is one of the most common complications of enteral nutrition [17]. In another study conducted by Xie et al. [18], the authors found that enteral nutrition accompanied by probiotics administered for 8 days to patients with gastric cancer not only reduced the incidence of diarrhea in the postoperative period $(p=0.002)$, but also enhanced the immune response. It was elegantly demonstrated that the levels of $\operatorname{IgG}, \operatorname{IgM}, \operatorname{IgA}$ increased, while the concentrations of proinflammatory cytokines such as IL-6, IL-8, TNF- $\alpha$ decreased. However, these results did not reach statistical power, probably due to the short period of the experimental phase (7-8 days).

Small intestinal bacterial overgrowth (SIBO) is diagnosed when there are 10 or more colony forming units (CFU) per $\mathrm{ml}$ in the proximal part of the small intestine. Its prevalence was estimated to vary between 2.5 and $22 \%$ in healthy adults (depending on e.g. body mass index) and $4-70 \%$ in patients with irritable bowel syndrome [27]. Small intestinal bacterial overgrowth may be associated with the presence of gastric and colorectal cancer. Probiotic therapy may counteract SIBO or diminish its severity. These hypotheses were proven by Liang et al., who analyzed 112 patients with gastric cancer and 88 diagnosed with colorectal cancer (CRC) [28]. In order to confirm $\mathrm{SIBO}$, the glucose-H2-breath test was performed. The gastrointestinal symptoms were assessed by a questionnaire, which included abdominal pain, bloating, constipation, appetite, diarrhea and fever. Patients in whom SIBO was confirmed $(63 \%)$ received the Bifidobacterium triple viable (Bifico) mixture containing different probiotic strains belonging to Enterococcus, Bifidobacteria and Lactobacillus genera or placebo. After the treatment $19 \%$ of patients 
receiving probiotic therapy were SIBO-positive whereas in the placebo group SIBO was confirmed in $74.6 \%$ $(p<0.01)$. These results confirmed that Bifidobacterium triple viable capsule therapy may be effective in combating SIBO. Moreover, the authors concluded that the presence of SIBO in cancer patients was associated with proton pump inhibitor usage and the administration of probiotic formula reduced gastrointestinal symptoms, so probably it may be useful in SIBO treatment [28].

\section{Colorectal cancer}

Currently, CRC is the third most common cancer worldwide. It was reported that the abundance of Enterococcus faecalis, Fusobacterium nucleatum, Streptococcus bovis/gallolyticus, Escherichia coli, Peptostreptococcus anaerobius, Bacteroides fragilis and Helicobacter hepaticus has an impact on colorectal carcinogenesis [20, 29-32]. Patients with CRC were shown to have higher counts of Enterococcus faecalis in comparison to healthy subjects [33]. Fusobacterium nucleatum was demonstrated to modulate the E-cadherin signaling pathway, cause the activation of T-cell factor, $\beta$-catenin, NF- $\kappa \mathrm{B}, \mathrm{c}-\mathrm{myc}$ and cyclin D1. These may enhance proliferation of colon cancer cells [34]. In Streptococcus bovis-infected tissues, higher expression levels of pro-inflammatory mediators, such as IL-1 $\beta$, IL-8 and COX-2, were found [35]. Helicobacter hepaticus causes the secretion of pro-inflammatory mediators, such as IL-1 $\beta$, IL-6, IL-8, TNF- $\alpha$, IFN- $\gamma$, so it also contributes to the development of inflammation-induced cancer. Due to the fact that Escherichia coli persists in immune cells and controls secretion of pro-tumoral mediators, it also has an impact on CRC progression [36]. Peptostreptococcus anaerobius abundance was found to be associated with the incidence of CRC in the mechanism encompassing interaction with TLR2 and TLR3, activation of SREBP-2 and AMPK signaling and inducing oxidative stress [29]. Bacteroides fragilis endotoxin was reported to elevate IL-11 production in colon cancer patients, whilst another toxin, fragilysin, was shown to play a role in E-cadherin cleavage, inducing the Th-17 response and activation of STAT3 and $\beta$-catenin signaling [30, 31]. Cytolethal distending toxin of Helicobacter hepaticus was found to increase the expression of $p 21$ and promote senescence and endoreplication, thus initiating giant polyploid cells in mice [32].

Porphyromonas gingivalis is a periopathogen involved in chronic periodontitis, which through interference with innate immunity promotes dysbiosis [37]. As stated above, this pathogen is associated with pancreatic cancer occurrence, but also it can promote the development of colorectal cancer. Porphyromonas spp. (primarily P. asaccharolytica) are among the most consistently enriched taxa in patients with colorectal cancers [38]. According to Wang et al., $P$. gingivalis regulates the NLRP3 inflammasome that results in the generation of a pro-inflammatory micro- environment which plays an important role in the progression of colorectal neoplasia [39]. On the other hand, the most recent study has shown that higher amounts of Fusobacterium nucleatum and Streptococcus gallolyticus, but not Porphyromonas gingivalis, are observed in the saliva of patients suffering from CRC in comparison to healthy subjects, $p=0.837$ [40].

The Wnt- $\beta$-catenin signaling pathway impacts many processes such as regulation, differentiation, proliferation and cellular death. Members of the Wnt signaling pathway and its inhibitors can be used as biomarkers for diagnosis and cancer treatment [41]. The Wnt/ $\beta$-catenin signaling pathway was described for the first time at the turn of the 1970s and 80s. This biochemical pathway has been divided into the canonical pathway and several noncanonical pathways. The canonical pathway has been linked to $\beta$-catenin. It regulates $\mathrm{T}$ cell transcription factors influencing embryogenesis, differentiation, survival and proliferation of cells. Dysfunction of the $\mathrm{Wnt} / \beta$-catenin signaling pathway is observed in several types of cancer [41]. Anticancer drugs have the ability to modulate the $\mathrm{Wnt} / \beta$-catenin signaling pathway, but the knowledge about the role of non-toxic agents in this field is insufficient. Probiotics-derived substances may be used as adjuvants in combination with drugs. Indeed, an in vitro study showed that Lactobacillus plantarum ATCC 10241-derived supernatant inhibited the specific markers of 5-florouracil (5-FU)-resistant CRC cells, namely CD44, 133, 166, and ALDH1, which are epitopes of cancer stem cells. Also, such co-therapy enhanced the efficacy of 5-FU via elevation of cancer cell death and apoptosis. Most importantly, the combination therapy elevated the inhibition of activated $\mathrm{Wnt} / \beta$-Catenin signaling in chemoresistant CRCs. The authors proved that probiotic bacteria supernatant normalized the expression of tankyrase 1 and axin- 2 in chemoresistant cells to levels typically observed in 5-FU non-resistant parenteral cells and consequently inhibited the accumulation of $\beta$-catenin in the nucleus. Finally, L. plantarum ATCC 10241 and 5 -FU reduced the formation and size of colonospheres [42]. However, the data on non-toxic agents which are $\mathrm{Wnt} / \beta$-catenin inhibitors are scarce.

In an animal study (colon cancer model) it was reported that administration of Lactobacillus acidophilus DSM 13241 and Bifidobacterium bifidum (both in a dose of 1 x $10 \mathrm{CFU} / \mathrm{g}$ ) for 5 months decreased expression of miR-135b, miR-155 and KRAS and increased expression of miR-26b, miR-18a, APC, PU.1 and PTEN [43]. These results confirmed potential capabilities of Lactobacillus plantarum and Bifidobacterium bifidum to increase the expression of the tumor suppressor miRNA and decrease the oncogenes linked to improved colon cancer treatment [43]. Another animal model demonstrated that administration of Clostridium butyricum reduced the counts of Th1 and Th17 lymphocytes and proinflammatory factors (NF-кB, IL-22), as well as supporting apoptosis of cancer cells [44]. 
Walia et al. in a study conducted in rats demonstrated that administration of probiotics suppressed the COX-2 expression, potentially providing protection against colon cancer [45]. Similar results were obtained by Kaeid Sharaf et al. The authors utilized an experimental colon carcinogenesis model and administration of Lactobacillus rhamnosus GG and Lactobacillus acidophilus NCDC15 in conjunction with celecoxib, an selective COX-2 inhibitor, to prove that such a combination elevated the efficacy and minimized therapy adverse effects. Rats administered the abovementioned therapeutics showed reduced aberrant crypt foci (ACF) count and expression of procarcinogenic molecular markers such as $\beta$-catenin, NF- $\mathrm{KB}$ and COX-2. These results - typically found in celecoxib treatment were however more pronounced when drugs were given conjointly with microbial agents [46]. Potential prevention of colon carcinogenesis by probiotics was also evaluated by Ohara et al., who analyzed 27 healthy persons. The participants were divided into 2 groups: consuming yogurt containing Bifidobacterium longum $(n=14)$ and taking yogurt containing $B$. longum and fructooligosaccharides $(n=13)$ once daily for 5 weeks. This regimen was associated with a higher Bifidobacterium detection rate compared to the study group. In addition, B. longum increased the total content of SCFAs in the feces and decreased the detection rate of Bacteroides fragilis and growth of putrefactive bacteria [47]. It is essential as B. fragilis is an opportunistic human pathogen, which may cause peritoneal infections [48].

Probiotics may potentially prevent postoperative infection and related complications in colorectal cancer. A meta-analysis conducted by Ouyang et al. demonstrated that probiotics may decrease infection rate, incision infection and pneumonia incidence by almost half. The first flatus time was found to be shortened significantly by probiotic administration [49]. Moreover, probiotics (Lactobacillus rhamnosus $G G$ ) may reduce the frequency of severe diarrhea and abdominal discomfort related to 5-FU based chemotherapy [50]. The administration of probiotic preparation VSL\#3 (L.casei, L. plantarum, L. acidophilus, L. delbrueckii, Bifidobacterium longum, B. breve, B. infantis and Streptococcus thermophilus) may reduce the risk of diarrhea after radiotherapy [51]. Importantly, as reported by Skonieczna-Żydecka et al., the efficacy of probiotics in counteracting surgery related complications has been linked to SCFAs synthesis [52].

\section{Pancreatic cancer}

The most common type of pancreatic cancer is pancreatic ductal adenocarcinoma (PDAC). Chronic pancreatitis, diabetes, cigarette smoking, and obesity are the risk factors for PDAC [53, 54]. Moreover, there are studies showing an association between oral, duodenal, intestinal dysbiosis and the PDAC occurrence.
The composition of gut microbiota in pancreatic cancer (PC) patients was proved to be altered. Reduced diversity and the higher abundance of certain pathogens and lipopolysaccharides-producing bacteria (such as Veillonella, Klebsiella, Selenomonas, and Prevotella, Hallella, Enterobacter), as well as diminished counts of beneficial Bifidobacterium, Coprococcus, Clostridium IV, Blautia, Flavonifractor, and Anaerostipes was a fecal microbiota fingerprint in PC [55]. In contrast, patients with the cancer in the head of the pancreas had similarly diverse microbiome in duodenal biopsy and significantly more diverse microbiome of the tongue coat in comparison with healthy individuals $[56,57]$. In a recent study, the authors demonstrated that bacteria from the duodenum migrated to the pancreas by means of bacterial DNA profile comparison between two organs [58]. Furthermore, patients with pancreatic cancers had lower relative abundance of Lactobacillus spp. and higher relative abundance of Fusobacterium spp. in pancreatic tissues in comparison to non-cancer individuals [58]. Additionally, DNA of Helicobacter spp. was repeatedly reported to be detected in pancreatic cancer tissues $[59,60]$. Moreover, presence of Fusobacterium spp. in PDAC tissue was independently associated with a worse prognosis [60]. The other studies proved that not only gut but also oral dysbiosis may also be associated with higher risk of pancreatic cancer. It was evidenced that increased counts of Porphyromonas gingivalis and elevated levels of blood serum antibodies against this bacterial species dominate in pancreatic cancer patients [61]. Other studies demonstrated that low concentrations of Neisseria elongata, Corynebacterium spp., and Streptococcus mitis and increased concentrations of Granulicatella adiacens and Porphyromonas gingivalis may indicate the cancer origin [62, 63].

Helicobacter pylori infection is also a considerable risk factor for PDAC and is thought to be involved in chronic and acute pancreatitis (as well as autoimmune pancreatitis). Pathogenic components (ammonia, LPS, inflammatory cytokines) derived from $H$. pylori cause pancreas damage. H. pylori infection may lead to dysregulation of cellular processes by activating NF- $\mathrm{NB}$ and AP-1 (activator protein-1). The increased level of IL- 8 accelerates the inflammation and consequently leads to pancreatic carcinogenesis. Such infection may lead to dysregulation of cellular processes by activating NF- $\mathrm{BB}$ and AP-1 (activator protein-1) [64]. In over $90 \%$ of cases of PDAC KRAS gene mutations have been observed [65]. As the pancreas does not contain its own microbiota, sterile inflammation within this organ may be triggered by LPS thus mediated through TLRs. Of note, LPS from Helicobacter pylori has the ability to hyperstimulate mutations of the KRAS gene and initiate the process of pancreatic carcinogenesis [66]. In fact, LPS binding to TLR4 was elegantly linked to chronic inflammation and tumorigenesis, overall [67]. A rodent study by Ochi et al. proved that LPS triggered the 
progression of pancreatic cancer, whilst the blockade of TLR4 slowed down the progression of the disease. On the other hand, TLR4 adapter protein MyD88 was found to be pro-cancerogenic via inducing pancreatic antigen-restricted Th2 cell by dendritic cells [68]. Moreover, Helicobacter pylori infection causes persistent STAT3 activation, and due to this reason it can promote pancreatic cancer progression by upregulating the expression of anti-apoptotic and pro-proliferative proteins (Bcl-xL, MCL-1, survivin, c-myc, cyclin D-1) [66, 69].

Of high importance, a drug commonly used in pancreatic cancer - gemcitabine - was found to be inactivated in the presence of some bacterial genera producing cytidine deaminase responsible for this process, as discussed later [70]. It therefore raises the question whether antibiotic treatment could somehow regulate the response to the treatment in this and other cancers, physiologically owing no microbiota.

Nomura et al. examined the effect of use of probiotic on frequency of postoperative infectious complications after pancreatoduodenectomy. There were 30 patients who received probiotic (Enterococcus faecalis, Clostridium butyricum, Bacillus mesentericus) and 34 who consumed placebo. The probiotics were administered immediately after admission, 3-15 days before the operation and on the second postoperative day. Postoperative infectious complications occurred in $23 \%$ of patients receiving probiotic and in $53 \%$ of patients consuming placebo $(p=0.02)$ [71]. Probiotic therapy seems to be useful in reduction of postoperative infectious complications in pancreatic cancer patients.

\section{Liver cancer}

Currently, liver cancer is the fifth most common cancer in men and the ninth in women [20]. The majority of primary liver cancers are hepatocellular carcinoma (HCC). The main factor contributing to the development of HCC is obesity, and thus also a high-fat diet. The gut microbiota is associated with development of HCC [72]. The most important changes in composition of the intestinal microbiota in cirrhosis comprise enrichment of Veillonella and Streptococcus spp. and lowered counts of Clostridiales [73]. Upper gastrointestinal tract bacterial overgrowth was linked to increased LPS levels circulating in blood [74]. At the same time, bacterial translocation in the upper gastrointestinal tract was found to be associated with the development of liver diseases [74]. In those cases modulation of microbiota by probiotics seems to be beneficial. An animal model study showed that the probiotic mixture Prohep (Lactobacillus rhamnosus GG, viable Escherichia coli Nissle 1917, heat-inactivated VSL\#3) significantly slowed down the tumor growth and reduced its size [75]. As HCC is a vascularized tumor, Th17/IL-17+ cells may play an important role in angiogenesis and progression of this carcinoma [75]. In a rat study, Kumar et al. reported that probiotics reduced the tumor incidence and decreased c-myc, bcl-2, cyclin D1, rasp-21 levels. As a consequence, probiotics potentially provided protection against AFB1-induced hepatocarcinogenesis [76]. In another animal study it was proved that VLS\#3 administration restores gut microbiome homeostasis and ameliorates intestinal and hepatic inflammation. It inhibited the progression of cirrhosis to HCC [77].

\section{The impact of gut microbiota on efficacy and safety of anticancer treatment}

The treatment of gastrointestinal cancers includes surgery, chemotherapeutics, and biological drugs [78]. The choice of appropriate care is determined by scientific guidelines, which are adjusted to clinical case and sociological and economical constraints [79]. Gut microbiota dysbiosis of a cancer patient has recently been considered as a prognostic factor of the treatment success [15]. Currently, at least a few studies on this issue have been published. Such a link has already been proven for immunotherapy, f.i. therapy with PD-1/PD-L1 antibodies mentioned before and is in the testing phase for capecitabine in CRC treatment [80]. Moreover, many cohort studies have shown that patients with CRC who have a large amount of DNA copies of Fusobacterium nucleatum (anaerobic bacteria typical for oral microbiota) in tumor biopsies live significantly shorter than patients with lower counts of this genus [81-88].

\section{Surgical treatment}

Surgical treatment - tumor resection with optional resection of surrounding tissues and lymph nodes - belongs to the basic anticancer therapy of gastrointestinal cancer. After resection of a part of the digestive tract it is necessary to reconstruct its continuity. One of the serious postoperative complications is anastomosis leakage, which is associated not only with the possibility of developing peritonitis, but also with higher risk of local tumor recurrence and a smaller percentage of five-year survival after the operation. The risk of anastomotic leak depends, among other factors, on the composition and activity of the gastrointestinal microbiota [86]. Preliminary research has demonstrated that patients with esophagectomy-type anastomotic leak have increased variance in their preoperative oral and gastric flora in comparison to patients without this condition [87]. Also, in patients developing such leak, within the intraoperative tissue, lower microbial diversity was detected as well as increased number of mucin-degrading members belonging to Lachnospiraceae (especially Blautia obeum) and Bacteroidaceae families [88]. It is believed that reduced microbial diversity predisposes to the development of different surgery related complications as a result of antibiotic therapy and the colonization of the intestinal microbiome by pathogens. Studies using animal 
models proved that Enterococcus faecalis and Pseudomonas aeruginosa contribute to the pathogenesis of anastomotic leak. Strains of these bacteria, which were isolated from leaking tissues, were shown to elevate production of collagenase, which breaks down collagen I and activates local tissue matrix metalloproteinase to finally cleave collagen IV and induce anastomotic damage [89, 90].

It was well established that the majority of hospital infections originate from the patient's own intestinal ecosystem following the deleterious therapy and thus physiological stress [91]. For instance, tissue ischemia during the surgical procedure may be partially responsible for the elevation of virulent Escherichia coli, Enterococcus faecalis and abovementioned Pseudomonas aeruginosa [91-93]. As a consequence of elevated intestinal permeability, gut antigens easily flow through the epithelium and serum inflammatory mediator concentrations may elevate and further precede bacterial translocation into mesenteric lymph nodes as demonstrated after hepatectomy and esophagectomy [94-96]. Thus probiotics, administered preoperatively via balancing the gut microbiota composition, may lower the risk of surgery related complications. SkoniecznaŻydecka et al. demonstrated recently that microbial agents ingested before the surgery increased the abundance of beneficial microbiota, which consequently improved the immune response secondary to elevated short chain fatty acid synthesis [46]. Importantly, studies that entered the meta-analysis were predominantly cancer-related [46].

\section{Chemotherapy}

Chemotherapy is an adjuvant treatment especially in the case of pancreatic and colorectal cancers. The most commonly used chemotherapeutics include fluorouracil, gemcitabine, capecitabine, oxaliplatin and irinotecan. Anticancer activity using fluorouracil (as well as its prodrug, capecitabine) and gemcitabine (pyrimidine antimetabolites) results from the disruption of DNA synthesis, DNA damage and ultimately induction of apoptosis in cancer cells. It was shown that worse prognosis for patients with high Fusobacterium nucleatum counts in the dissected colon cancer tissue may be due to induction of fluorouracil chemoresistance by this bacterium [97]. Fusobacterium nucleatum stimulates cancer cells to autophagy, preventing apoptosis of tumor cells [97], and increases the amount of BIRC3 - a protein directly inhibiting apoptosis by binding caspases [84]. Another mechanism behind chemoresistance is linked to gemcitabine used to treat pancreatic cancer. Some intestinal bacteria, mainly belonging to the Gammaproteobacteria class, express a long isoform of cytidine deaminase, which can break down gemcitabine into an inactive metabolite (2',2'-difluorodeoxyuridine). It turned out that up to three-quarters of the PDAC contain bacteria in their structure, and more than half of the identified bacteria belong to Gammaproteobacteria (mainly Enterobacteriaceae and Pseudomonadaceae families) [98].
It has been shown that bacteria isolated from human PDACs provide resistance to gemcitabine for in vitro culture of CRC cells, suggesting that anti-bacterial therapy should be considered in the treatment of gemcitabine in patients with PDAC [99]. The activity of gastrointestinal microbiota may have an impact on the development of complications in the course of chemotherapy. It has been shown that patients with colorectal cancer, whose intestinal microbiota produces methane, are significantly more likely to experience constipation as a result of fluorouracil treatment (OR $=2.23,95 \%$ CI: 1.35-3.68, $p=0.002)$ [100]. Currently, research is underway to use the composition of intestinal microbiota as a predictor of response to therapy, for example capecitabine in CRC [79].

Oxaliplatin exhibits its anti-tumor activity primarily by inhibiting DNA replication as a result of intra- and inter-chain cross-links in DNA. However, its ability to induce the formation of oxygen radicals (ROS) that damage DNA and induce tumor cell apoptosis has also been described [101]. Similarly to fluorouracil, Fusobacterium nucleatum has been shown to induce chemoresistance of tumor cells to oxaliplatin by activating autophagy in them [97]. On the other hand, animal models have shown that the use of antibiotics limits the antitumor effect of oxaliplatin by inhibiting tumor infiltration by ROS-producing leukocytes [102].

\section{Immunotherapy}

The composition of the intestinal microbiome is an independent predictor of the response and efficacy of cancer immunotherapy. Routy et al. found that patients with cancer treated with anti-PD-1/PD-L1 antibodies lived significantly shorter if they took oral antibiotics during the immunotherapy period [103]. Further studies showed that the response to therapy correlated with the percentage of Akkermansia muciniphila in the microbiome of patients' feces. Importantly, both the transplantation of microbiota from patients responding to therapy and the supplementation A. muciniphila alone restored the sensitivity to immunotherapy in animals insensitive to immunotherapy due to antibiotic therapy [103]. On the other hand, it seems that immunotherapy modifies the composition of the microbiome, and the first case study indicated that fecal transplantation may be an effective method for treating intestinal inflammation caused by cancer immunotherapy [104].

\section{Impact of gastrointestinal cancer treatment on gut microbiota}

The effect of total and partial gastrectomy on the composition of the intestinal microbiome in patients treated for gastric cancer is the subject of debate in past and ongoing clinical trials [105]. More data on the effects of different treatment patterns in CRC and their consequences for the composition of intestinal microbiota was evidenced. There were significant differences in the gut microbiome of pa- 
Table 1. Meta-analyses of the efficacy of probiotics counteracting intestinal barrier disruptions secondary to cancer treatment

\begin{tabular}{|c|c|c|c|c|}
\hline Study aim & $\begin{array}{c}\text { Number } \\
\text { of trials/patients }\end{array}$ & $\begin{array}{l}\text { Primary } \\
\text { diagnosis }\end{array}$ & Outcome & Reference \\
\hline $\begin{array}{l}\text { To evaluate the efficacy } \\
\text { of probiotics on the intestinal } \\
\text { mucosa barrier indices }\end{array}$ & $17 / 1242$ & $\begin{array}{l}\text { Colorectal } \\
\text { cancer }\end{array}$ & $\begin{array}{c}\text { Probiotics significantly affect } \\
\text { L/M ratio: } \mathrm{SMD}=3.83, p=0.001 \\
\text { Bifidobacterium/Escherichia ratio }(\mathrm{SMD}=3.91, p=0.000) \\
\text { Ocludin ratio }(\mathrm{SMD}=4.74, p=0.000) \\
\text { Bacterial translocation }(\mathrm{SMD}=3.12, p=0.002) \\
\text { SIgA level }(\mathrm{SMD}=2.91, p=0.004) \\
\text { CRP level }(\mathrm{SMD}=4.21, p=0.000) \\
\text { - probiotics do not influence } \\
\text { IL-6 level }(\mathrm{SMD}=1.33, p=0.184)\end{array}$ & [115] \\
\hline $\begin{array}{l}\text { To assess whether probiotics } \\
\text { may prevent cancer } \\
\text { therapy-induced diarrhea }\end{array}$ & 7/1091 & & $\begin{array}{l}\text { Probiotics do not prevent or reduce the overall incidence } \\
\text { of diarrhea (RR }=0.81,95 \% \text { CI: } 0.60-1.09, p=0.16)\end{array}$ & [116] \\
\hline $\begin{array}{l}\text { To establish a link between } \\
\text { probiotics and the incidence } \\
\text { of cancer surgical infection }\end{array}$ & $7 / 816$ & $\begin{array}{l}\text { Colorectal } \\
\text { cancer }\end{array}$ & $\begin{array}{l}\text { Probiotics significantly affected: } \\
\text { bacteria in blood: } \mathrm{OR}=0.4069 \text { (95\% CI: } 0.2662-0.6222 \\
p<0.0001 \text { ) infectious complications: } \mathrm{OR}=0.5388 \\
(95 \% \text { CI: } 0.4058-0.7154, p<0.0001)\end{array}$ & [117] \\
\hline \multirow{2}{*}{$\begin{array}{l}\text { To assess whether probiotics } \\
\text { may support } H \text {. pylori } \\
\text { eradication }\end{array}$} & $14 / 1671$ & $\begin{array}{l}\text { H. pylori } \\
\text { infection }\end{array}$ & $\begin{array}{l}\text { Probiotic therapy significantly increases rate of } H \text {. pylori } \\
\text { eradication: OR }=1.84 \text { (95\% CI: } 1.34-2.54)\end{array}$ & [118] \\
\hline & $30 / 4302$ & $\begin{array}{l}\text { H. pylori } \\
\text { infection }\end{array}$ & $\begin{array}{l}\text { Probiotics significantly improve } H . \text { pylori eradication rates } \\
\text { (APP: } \mathrm{RR}=1.122 ; 95 \% \text { CI: } 1.091-1.153 ; p<0.001 \text {; } \\
\text { ITT: } \mathrm{RR}=1.141 ; 95 \% \text { CI: } 1.106-1.175 ; p<0.001 \text { ) }\end{array}$ & [119] \\
\hline
\end{tabular}

$S M D$ - standardized mean difference, ITT - intention to treat, APP - as-per-protocol, L/M - lactulose/mannitol, RR - relative risk, OR - odds ratio, CI - confidence interval, CRP - C-reactive protein, H. pylori-Helicobacter pylori

tients who underwent surgery without chemotherapy and people treated with a set of chemotherapeutics (oxaliplatin and tegafur - a fluorouracil precursor). Patients treated surgically had a smaller number of Bacteroidetes and Firmicutes, and thus less biodiverse microbiota, while patients treated with chemotherapeutics were the only ones who had Veillonella dispar and significantly higher Prevotellacopri and Bacteroides plebeius counts than in the other groups [106]. Unfortunately, the authors of the studies concerning changes in the human gut microbiome after anti-cancer treatment have not investigated the clinical value of the observed changes. Instead, enriched bacterial strains or genera have been called putative biomarkers of response to anti-cancer treatments. However, the authors have not provided data about chemotherapy responsiveness or clinical outcomes of patients. Hence, we did not call them biomarkers in our manuscript. It would be valuable to investigate the correlations between enriched bacterial strains or genera and (1) occurrence of side-effects of chemotherapy and (2) survival time/5-year survival rate [106]. More valuable longitudinal examination - comparing the microbiome of the same patients before and on average one month after surgical treatment of rectal cancer - confirmed the biodiversity decline of the microbiome as a result of surgery. The decrease of Bacteroidetes, Roseburia, Alistipes, Clostridium XlVa, Fusobacterium, Parvimonas, Peptostreptococcus and increase of Klebsiella bacteria were reported [107]. However, in a study in which the composition of the microbiome was analyzed, a year after the treatment of colorectal cancer, normalization of the microbiome was observed - regardless of the therapeutic procedure [107]. The transient decrease in diversity of gut microbiome can free up previously unavailable ecological niches, and thus can promote colonization or growth of pathogens [108], i.e. can be responsible for the increased risk of Clostridium difficile infection in post-surgery CRC patients. Indeed, on the one hand, a number of studies and models have shown the association between decrease in diversity and higher risk of $C$. difficile colonization [109] or higher risk of $C$. difficile recurrence [110]. On the other hand, $C$. difficile infection is a known complication of colorectal surgery that increases perioperative morbidity and mortality; it affects up to $4.2-6.8 \%$ of post-surgery CRC patients $[111,112]$. Changes in the composition of intestinal microbiome during treatment with fluorouracil and irinotecan are probably secondary to intestinal epithelial damage, as indicated by the observed sequence of disorders in the mouse mucosal inflammatory model of fluorouracil [113] and the neglected effect of fluorouracil and irinotecan on the composition and function of intestinal microbiota in vitro [114].

As demonstrated by a few meta-analyses, probiotics should be taken into account during and after cancer treatment. It was proved that at least some of the unpleasant 
symptoms that developed secondary to cancer treatment may be counteracted by probiotics. Most importantly, the markers of gut barrier integrity were shown to be at least partially improved with such intervention [115-119]. A summary is presented in Table 1.

\section{Conclusions}

Extensive studies provided evidence on the involvement of the human microbiome in regulating multiple signaling pathways linked to carcinogenesis. Moreover, a battery of bacterial enzymes was shown to affect xenobiotic metabolism and thus to potentially modulate cancer therapy effectiveness. The administration of probiotic bacteria seems to be useful in supporting treatment of patients suffering from cancer diseases, mainly due to their immunomodulatory properties and the ability to reduce side effects of anti-cancer therapy. Future analyses are on the horizon to elucidate whether the microbiome may serve as a basis for diagnostic and treatment approaches in cancer medicine.

\section{The authors declare no conflict of interest.}

\section{References}

1. Valdes AM, Walter J, Segal E, et al. (2018): Role of the gut microbiota in nutrition and health. Br Med J 361: k2179.

2. Martin R, Bermundez-Humaran LG, Langella P (2016): Gnotobiotic rodent: an in vivo model for the study of microbe-microbe interactions. Front Microbiol 7: 409.

3. Erny D, de Angelis H, Prinz M (2017): Communicating systems in the body: how microbiota and microglia cooperate. Immunology 1: 7-15.

4. Rosshart SP, Vassallo BG, Angelleti D, et al. (2017): Wild mouse gut microbiota promotes host fitness and improve disease resistance. Cell 171: 1015-1028.

5. Dzutsev A, Badger JH, Perez-Chanona E, et al. (2017): Microbes and cancer. Ann Rev Immunol 35: 199-228.

6. Goodman B, Gardner H (2018): The microbiome and cancer. J Pathol 5: 667-676.

7. Paulos CM, Wrzesinski C, Kaiser A, et al. (2007): Microbial translocation augments the function of adoptively transferred self/tumor-specific CD8+ T cells via TLR4 signaling. J Clin Invest 117: 2197-2204.

8. Lida N, Dzutsev A, Stewart CA, et al. (2013): Commensal bacteria control cancer response to therapy by modulating the tumor microenvironment. Science 342: 967-970.

9. Daillere R, Vetizou M, Waldschmitt N, et al. (2016): Enterococcus hirae and Barnesiellaintestinihominis facilitate cyclophosphamide-induced therapeutic immunomodulatory effects. Immunity 45: 931-943.

10. York A (2018): Gut microbiota sways response to cancer immunotherapy. Nat Rev Microbiol 16: 21.

11. Vetizou M, Pitt JM, Daillere R, et al. (2015): Anticancer immunotherapy by CTLA-4 blockade relies on the gut microbiota. Science 350: 1079-1084.

12. Routy B, le Chatelier E, DerosaL, et al. (2018): Gut microbiome influences efficacy of PD-1 based immunotherapy against epithelial tumors. Science 359: 91-97.
13. Sivan A, Corrales L, Hubert N, et al. (2015): Commensal Bifidobacterium promotes antitumor immunity and facilitates anti-PD-L1 efficacy. Science 350: 1084-1089.

14. Matson V, Fessler J, Bao R, et al. (2018): The commensal microbiome is associated with anti-PD-1 efficacy in metastatic melanoma. Science 359: 104-108.

15. Zitvogel L, Ma Y, Raoult D, et al. (2018): The microbiome in cancer immunotherapy: diagnostic tools and therapeutic strategies. Science 359: 1366-1370.

16. Tong JL, Ran ZH, Shen J, et al. (2007): Meta-analysis: the effect of supplementation with probiotics on eradication rates and adverse events during Helicobacter pylori eradication therapy. Aliment Pharmacol Ther 25: 155-168.

17. Zhao R, Wang Y, Huang Y, et al. (2017): Effects of fiber and probiotics on diarrhea associated with enteral nutrition in gastric cancer patients. Medcinie 96: e8418.

18. Xie H, Lu Q, Wang H, et al. (2018): Effects of probiotics combined with enteral nutrition on immune function and inflammatory response in postoperative patients with gastric cancer. J Buon 23: 678-683.

19. Floch P, Megraud F, Lehours P (2017): Helicobacter pylori strains and gastric MALT lymphoma. Toxins 9: 132.

20. Malfertheiner P, Megraud F, O'Morain CA, et al. (2017): Management of Helicobacter pylori infection - the Maastricht V/Florence Consensus Report. Gut 66: 6-30.

21. Wang L, Zhou J, Xin Y, et al. (2016): Bacterial overgrowth and diversification of microbiota in gastric cancer. Eur J Gastroenterol Hepatol 3: 262-266.

22. Yu G, Hu N, Wang L, al. (2017): Gastric microbiota features associated with cancer risk factors and clinical outcomes: a pilot study in gastric cardia cancer patients from Shanxi China. Int J Cancer 1: 45-51.

23. Javanmard A, Ashtari S, Sabet B, et al. (2018): Probiotics and their role in gastrointestinal cancer prevention and treatment; an overview. Gastroenterol Hepatol Bed Bench 11: 284-295.

24. Losurdo G, Cubisino R, Barone M, et al. (2018): Probiotic monotherapy and Helicobacter pylori eradication: a systematic review and pooled-data analysis. World J Gastroenterol 24: 139-149.

25. Castano-Rodriquez N, Goh KL, Fock KM, et al. (2017): Dysbiosis of the microbiome in gastric cancer. Sci Rep 7: 15957.

26. Kłęk S, Jankowski M, Kruszewski WJ, et al. (2015): Standardy leczenia żywieniowego w onkologii. J Oncol 65: 320337.

27. Gulbicka K, Grzymisławski M (2016): Wzdęcia brzucha przyczyny i postępowanie. Piel Zdr Publ 6: 67-76.

28. 28. Liang S, Xu L, Zhang D, et al. (2016): Effect of probiotics on small intestinal bacterial overgrowth in patients with gastric and colorectal cancer. Turk J Gastroenterol 27: 227-232.

29. Tsoi H, Chu ESH, Zhang X, et al. (2017): Peptostreptococcus anaerobius induces intracellular cholesterol biosynthesis in colon cells to induce proliferation and causes dysplasia in mice. Gastroenterology 152: 1419-1433.

30. Ulger Toprak N, Yagci A, Gulluoglu BM, et al. (2006): A possible role of Bacteroides fragilis enterotoxin in the aetiology of colorectal cancer. Clin Microbiol Infect 12: $782-786$.

31. Sears CL, Islam S, Saha A, et al. (2008): Association of enterotoxigenic Bacteroides fragilis infection with inflammatory diarrhea. Clin Infect Dis 47: 797-803. 
32. Péré-Védrenne C, Prochazkova-Carlotti M, Rousseau B, et al. (2017): The cytolethal distending toxin subunit CdtB of helicobacter hepaticus promotes senescenceand endoreplication in xenograft mouse models of hepatic and intestinal celllines. Front Cell Infect Microbiol 7: 268.

33. Balamurugan R, Rajendiran E, George S, et al. (2008): Real-time polymerase chain reaction quantification of specific butyrate-producing bacteria, Desulfovibrio and Enterococcus faecalis in the feces of patients with colorectal cancer. J Gastroenterol Hepatol 23: 1298-1303.

34. Rubinstein MR, Wang X, Liu W, et al. (2013): Fusobacterium nucleatum promotes colorectal carcinogenesis by modulating E-cadherin/ $\beta$-catenin signaling via its FadA adhesin. Cell Host Microbe 14: 195-206.

35. Abdulamir AS, Hafidh RR, Bakar FA (2010): Molecular detection, quantification, and isolation of Streptococcus gallolyticus bacteria colonizing colorectal tumors: inflammation-driven potential of carcinogenesis via IL-1, COX-2, and IL-8. Mol Cancer 9: 249.

36. Raisch J, Rolhion N, Dubois A, et al. (2015): Intracellular colon cancer-associated Escherichia coli promote protumoral activities of human macrophages by inducing sustained COX-2 expression. Lab Invest 95: 296-307.

37. Olsen I, Lambris JD, Hajishengallis G (2017): Porphyromonas gingivalis disturbs host-commensal homeostasis by changing complement function. J Oral Microbiol 1: 1340085

38. Flynn KJ, Baxter NT, Schloss PD (2016): Metabolic and community synergy of oral bacteria in colorectal cancer. MSphere 3: e00102-16.

39. Wang Z, Wang X, Jia Y (2019): Porphyromonas gingivalis promotes colorectal cancer development by regulating NLRP3 inflammasome signaling. AACR Annual Meeting 13: 2358.

40. Guven DC, Dizdar O, Alp A, et al. (2019): Analysis of Fusobacterium nucleatum and Streptococcus gallolyticus in saliva of colorectal cancer patients. Biomark Med 9: 725-735.

41. Koziński K, Dobrzyń A (2013): Szlak sygnałowy Wnt i jego rola w regulacji metabolizmu komórki. Post Hig Med Dosw 67: 1098-1108.

42. An J, Ha EM (2016): Combination therapy of Lactobacillus plantarum Supernatant and 5-fluouracil increases chemosensitivity in colorecal cancer cells. J Microbiol Biotechnol 26: 1490-1453.

43. Heydari Z, Rahaie M, Alizadeh AM, et al. (2018): Effects of Lactobacillus acidophilus and Bifidobacterium bifidum probiotics on the expression of MicroRNA 136b, 26b, 18a and 155, and their involving genes in mice colon cancer. Probiotics Antimicrob Proteins 11: 1155-1162.

44. Chen ZF, Ai LY, Wang JL, et al. (2015): Probiotics Clostridium butyricum and Bacillus subtilis ameliorate intestinal tumorigenesis. Future Microbiol 10: 1433-1445.

45. Walia S, Kamal R, Kanwar SS, et al. (2015): Cyclooxygenase as a target in chemoprevention by probiotics during 1,2-dimethylhydrazine induced colon carcinogenesis in rats. Nutr Cancer 67: 603-611.

46. Kaeid Sharaf L, Shukla G (2018): Probiotics (Lactobacillus acidophilus and Lactobacillus rhamnosus GG) in conjunction with celecoxib (selective COX-2 inhibitor) modulated DMH- induced early experimental colon carcinogenesis. Nutr Cancer 70: 946-955.
47. Ohara T, Suzatani T (2018): Intake of Bifidobacterium longum and fructo-oligosaccharides prevents colorectal cancer. Euroasian J Hepatogastroenterol 8: 11-17.

48. Niestępski S, Harnisz M, Korzeniewska E, et al. (2017): Bacteroidesspp - znaczenie kliniczne, lekooporność i metody jej oznaczania. Post Mikrobiol 56: 67-76.

49. Ouyang X, Li Q, Shi M, et al. (2018): Probiotics for preventing postoperative infection in colorectal cancer patients: a systematic review and meta-analysis. Int J Colorectal Dis 34: 459-469.

50. Osterlund P, Ruotsalainen T, KorpelaR, et al. (2007): Lactobacillus supplementation for diarrhoea related to chemotherapy of colorectal cancer: a randomised study. Br J Cancer 97: 1028-1034.

51. Delia P, Sansotta G, Donato V, et al. (2007): Use of probiotics for prevention of radiation-induced diarrhea. World J Gastroenterol 13: 912-915.

52. Skonieczna-Żydecka K, Kaczmarczyk M, Łoniewski I, et al. (2018): A systematic review, meta-analysis, and metaregression evaluating the efficacy and mechanisms of action of probiotics and synbiotics in the prevention of surgical site infections and surgery-related complications. J Clin Med 7: 56.

53. Meng Ch, Bai Ch, Brown TD, et al. (2018): Human gut microbiota and gastrointestinal cancer. Genomics Proteomics Bioinformatics 16: 33-49.

54. Rabelo-Goncalves EM, Roesler BM, Zeitune JM (2015): Extragastric manifestations of Helicobacter pylori infection: possible role of bacterium in liver and pancreas diseases. World J Hepatol 7: 2968-2979.

55. Ren Z, Jiang J, Xie H et al. (2017): Gut microbial profile analysis by MiSeq sequencing of pancreatic carcinoma patients in China. Oncotarget 8: 95176-95191.

56. Mei QY, Huang Ch, Luo SZ, et al. (2018): Characterization of duodenal bacterial microbiota in patients with pancreatic head cancer vs. healthy controls. Pancreatology 18: 438-445.

57. Lu H, Ren Z, Li A, et al. (2019): Tongue coating microbiome data distinguish patients with pancreatic head cancer from healthy controls. J Oral Microbiol 11: 1563409.

58. del Castillo E, Meier R, Chuang M, et al. (2019): The microbiomes of pancreatic and duodenum tissues overlap and are higly subject specific but differ between pancreatic cancer and noncancer subjects. Cancer Epidemiol Biomarkers Prev 28: 370-383.

59. Nilsson H, Stenram U, Ihse I, et al. (2006): Helicobacter species ribosomal DNA in the pancreas, stomach and dudenum of pancreatic cancer patients. World J Gastroenterol 12: 3038-3043.

60. Mitsuhashi K, Nosho K, Sukawa Y, et al. (2015): Association of Fusobacterium species in pancreatic cancer tissues with molecular features and prognosis. Oncotarget 6: 7209-7220.

61. Fukuda A, Wang SC, Morris JP (2011): Stat3 and MMP7 contribute to pancreatic ductal adenocarcinoma initiation and progression. Cancer Cell 19: 441-455.

62. Ertz-Archambault N, Keim P, von Hoff D (2017): Microbiome and pancreatic cancer: a comprehensive topic review of literature. World J Gastroenterol 23: 1899-1908.

63. Farrell JJ, Zhang L, Zhou H, et al. (2012): Variations of oral microbiota are associated with pancreatic diseases including pancreatic cancer. Gut 61: 582-588.

64. Takayama S, Takahashi H, Matsuo Y (2007): Effects of Helicobacter pylori infection on human pancreatic cancer cell line. Hepatogastroenterology 54: 2387-2391. 
65. di Magliano MP, Logsdon CD (2013): Roles for KRAS in pancreatic tumor development and progression. Gastroenterology 144: 1220-1229.

66. Huang H, Daniluk J, Liu Y, et al. (2014): Oncogenic K-Ras requires activation for enhanced activity. Oncogene 33: 532535.

67. Kojima M, Morisaki T, Izuhara K, et al. (2000): Lipopolysaccharide increases cyclo-oxygenase- 2 expression in a colon carcinoma cell line through nuclear factor- $\mathrm{\kappa B}$ activation. Oncogene 19: 1225-1231.

68. Ochi A, Nguyen AH, Bedrosian AS, et al. (2012): MyD88 inhibition amplifies dendritic cell capacity to promote pancreatic carcinogenesis via Th2 cells. J Exp Med 209: 16711687.

69. Yu H, Pardoll D, Jove R (2009): STATs in cancer inflammation and immunity: a leading role for STAT3. Nat Rev Cancer 9: 798-809.

70. Voorde JV, Sabuncuoglu S, Noppen S, et al. (2014): Nucleoside-catabolizng enzymes in mycoplasma-infected tumor cell cultures compromise the cytostatic acitivity of the anticancer drug gemcitabine. J Biol Chem 289: 13054-13065.

71. Nomura T, Tsuchiya Y, Nashimoto A, et al. (2007): Probiotics reduce infectious complications after pancreatoduodenectomy. Hepatogastroenterology 57: 661-663.

72. Brandi G, de Lorenzo S, Candela M, et al. (2017): Microbiota, NASH, HCC and the potential role of probiotics. Carcinogenesis 38: 231-240.

73. Yu L, Schwabe RF (2017): The gut microbiome and liver cancer: mechanism and clinical translation. Nat Rev Gastroenterol Hepatol 9: 527-536.

74. Li J, Sung CYJ, Lee N, et al. (2016): Probiotics modulated gut microbiota suppresses hepatocellular carcinoma growth in mice. Proc Natl Acad Sci USA 113: e1306-e1314.

75. LX Y, Schwabe RF (2017): The gut microbiome and liver cancer: mechanisms and clinical translation. Nat Rev Gastroenterol Hepatol 14: 527-539.

76. Kumar M, Verma V, Nagpal R, et al. (2011): Effect of probiotic fermented milk and chlorophyllin on gene expressions and genotoxicity during AFB1-induced hepatocellular carcinoma. Gene 490: 54-59.

77. Zhang HL, Yu LX, Yang W, et al. (2012): Profound impact of gut homeostasis on chemically-induced pro-tumorigenic inflammation and hepatocarcinogenesis in rats. J Hepatol 57: 803-812.

78. Available from: https://www.mp.pl/pacjent/onkologia/leczenie/84482,leczenie-systemowe.

79. Available from: http://onkologia.zalecenia.med.pl/.

80. Aarnoutse R, de Vos-Geelen JMPGM, Penders J, et al. (2017): Study protocol on the role of inestinal microbiota in colorectal cancer treatment: a pathway to personalized medicine 2.0. Int J Colorectal Dis 32: 1077-1084.

81. Flanagan L, Schmid J, Ebert M, et al. (2014): Fusobacterium nucleatum associates with stages of colorectal neoplasia development, colorectal cancer, and disease outcome. Eur J Clin Microbiol 33: 1381-1390.

82. Wei Z, Shougen C, Shanglong L, et al. (2016): Could gut microbiota serve as prognostic biomarker associated with colorectal cancer patients' survival? A pilot study on relevant mechanism. Oncotarget 7: 46158-46172.

83. Sun Y, An Q, Tian X, et al. (2016): Fusobacterium nukleatum infection is correlated with tumor metastasis and postoperative survival colorectal cancer patients in China. Trans Cancer Res 5: 579-588.
84. Lee D, Han S, Kang J, et al. (2018): Association between Fusobacterium nucleatum, pathway mutation, andpatients prognosis in colorectal cancer. Ann Surg Oncol 25: 33893395.

85. Zhang S, Yang Y, Weng W, el al. (2019): Fusobacterium nucleatum promotes chemoresistance to 5 -fluorouracil by upregulation of BIRC 3 expression in colorectal cancer. J Exp Clin Canc Res 38: 14.

86. Gershuni VG, Friedman ES (2019): The microbiome-host interaction as a potential driver of anastomotic leak. Curr Gastroenterol Rep 21: 4.

87. Reddy RM, Weir WB, Barnett S, et al. (2018): Increased variance in oral and gastric microbiome correlates with esophagectomy anastomic leak. Ann Thorac Surg 105: 865-870.

88. van Praagh J, de Goffau M, Bakker I, et al. (2019): Mucus microbiome of anastomotic tissue during surgery has predictive value for colorectal cancer anastomotic leakage. Ann Surg 269: 911-916.

89. Shogan BD, Belogortseva N, Luong PM, et al. (2015): Collagen degradation and MMP9 activation by Enterococcus faecalis contributes to intestinal anastomotic leak. Sci Trasl Med 7: 286 ra68.

90. Guyton K, Alverdy JC (2017): The gut microbiota and gastrointestinal surgery. Nat Rev Gastroenterol Hepatol 14: 43-54.

91. Bachmann R, Leonard D, Delzenne N, et al. (2017): Novel insight into the role of microbiota in colorectal surgery. Gut 66: 738-749.

92. Hartman AL, Lough DM, Barupal DK, et al. (2009): Human gut microbiome adopts an alternative state following small bowel transplantation. Proc. Natl Acad Sci USA 106: 17187-17192.

93. Shimizu K, Ogura H, Asahara T, et al. (2015): Gut microbiota and environment in patients with major burns - a preliminary report. Burns 41: 28-33.

94. Usami M, Miyoshi M, Kanbara Y, et al. (2011): Effects of perioperative synbiotic treatment on infectious complications, intestinal integrity, and fecal flora and organic acids in hepatic surgery with or without cirrhosis. J Parenteral Enteral Nutr 35: 317-28.

95. Mizuno T, Yokoyama Y, Nishio H, et al. (2010): Intraoperative bacterial translocation detected by bacterium-specific ribosomal rna-targeted reverse-transcriptase polymerase chain reaction for the mesenteric lymph node strongly predicts postoperative infectious complications after major hepatectomy for biliary malignancies. Ann Surg 252: 1013-1019.

96. Nishigaki E, Abe T, Yokoyama Y, et al. (2014): The detection of intraoperative bacterial translocation in the mesenteric lymph nodes is useful in predicting patients at high risk for postoperative infectious complications after esophagectomy. Ann Surg 259: 477-84.

97. Yu T, Guo F, Yu Y, et al. (2017): Fusobacterium nucleatum promotes chemoresistance to colorectal cancer by modulating autophagy. Cell 170: 548-563.

98. Geller L, Barzliy-Rokni M, Danino T, et al. (2017): Potential role of intratumor bacteria in mediating tumor resistance to the chemotherapeutic drug gemcitabine. Science 357: 15561160.

99. Nomura T, Tsuchiya Y, Nashimoto A, et al. (2007): Probiotics reduce infectious complications after pancreatoduodenectomy. Hepatogastroenterology 57: 661-663.

100. Holma R, Korpela R, Sairanen U, et al. (2013): Colonic methane production modifies gastrointestinal toxicity asso- 
ciated with adjuvant 5-fluorouracil chemotherapy for colorectal cancer. J Clin Gastroenterol 47: 45-51.

101. Martinez-Balibrea E, Martinez-Cardus A, Gines A, et al. (2015): Tumor-related molecular mechanisms of oxaliplatin resistance. Mol Cancer Ther 14: 1767-1776.

102. Lida N, Dzutsev A, Stewart C, et al. (2013): Commensal bacteria control cancer response to therapy by modulating the tumor microenvironment. Science 349: 967-970.

103. Routy B, le Chatelier E, Derosa L, et al. (2018): Gut microbiome influences efficacy of PD-1 based immunotherapy against epithelial tumors. Science 359: 91-97.

104. Deng X, Li Z, Li G, et al. (2018): Comprison of microbiota in patients treated by surgery or chemotherapy by $16 \mathrm{~S}$ rRNA sequencing reveals potiental biomarkers for colorectal cancer therapy. Front Microbiol 9: 1607.

105. Available from: https://clinicaltrials.gov/ct2/show/NCT0 3418428.

106. Cong J, Zhu H, Liu D, et al. (2018): A pilot study: changes of gut microbiota in post-surgery colorectal cancer patients. Front Microbiol 9: 2777.

107. Sze MA, Baxter NT, Ruffin MT, et al. (2017): Normalization of the microbiota in patients after treatment for colonic lesions. Microbiome 5: 150.

108. Britton RA, Young VB (2014): Role of the Intestinal Microbiota in Resistance to Colonization by Clostridium difficle. Gastoenterol 6: 1547-1553.

109. Chen LA, Hourigan SK, Grigoryan Z, et al. (2019): Decreased fecal bacterial diversity and altered microbiome in children colonized with clostridium difficile. J Pedriatr Gastroenterol Nutr 4: 502-508.

110. Chang YJ, Antonopoulos DA, Kalra A, et al. (2008): Decreased diversity of the fecal microbiome in recurrent clostridium difficile - associated diarrhea. J Infect Dis 3: 435-438.

111. Wren SM, Ahmed N, Jamal A, et al. (2005): Preoperative oral antibiotics in colorectal surgery increase the rate of clostridium difficile colitis. Arch Surg 8: 752-756.

112. Yeom CH, Cho MM, Baek SK, et al. (2010): Risk factors for the development of clostridium difficile-associated colitis after colorectal cancer surgery. J Korean Soc Coloproctol 5: 329-333.

113. Hamouda N, Sano T, Oikawa Y, et al. (2017): Apoptosis, dysbiosis and expression of inflammatory cytokies and sequential events in the development of 5-fluorouracil-induced intestinal mucostitis in mice. Basic Clin PharmacolToxicol 121: 159-168.

114. Vanlancker E, Vanhoecke B, Stringer A, et al. (2017): 5-fluorouracil and irinotecan (SN-38) have limited impact on colon microbial functionality and composition in vitro. Peer J 5: e 4017.

115. Liu D, Jiang XY, Zhou LS, et al. (2016): Effects of probiotics on intestinal mucosa barrier in patients with colorectal cancer after operation: meta-analysis of randomized controlled trials. Medicinie 95: e3342.

116. Wardill HR, van Sebille YZA, Ciorba MA, et al. (2018): Prophylactic probiotics for cancer therapy-induced diarrhoea: a meta-analysis. Curr Opin Support Palliat Care 12: 187-197.

117. de Andrade Calaca PR, Bezzerra RP, Campos Albuquerque WW, et al. (2017): Probiotics as preventive strategy for surgical infection in colorectal cancer patients: a systematic review and meta-analysis of randomized trials. Transl Gastroenterol Hepatol 2: 67.
118. Tong JL, Ran ZH, Shen J, et al. (2007): Meta-analysis: the effect of supplementation with probiotics on eradication rates and adverse events during Helicobacter pylori eradication therapy. Aliment Pharmacol Ther 25: 155-168.

119. Lau CS, Ward A, Chamberlain RS (2016): Probiotics improve the efficacy of standard triple therapy in the eradication of Helicobacter pylori: a meta-analysis. Infect Drug Resist 9: 275-289. 